\title{
A note on approximation of plurisubharmonic functions
}

\author{
Håkan Persson and Jan Wiegerinck
}

Dedicated to John Erik Fornæss on occasion of his 70th birthday

\begin{abstract}
We extend a recent result of Avelin, Hed, and Persson about approximation of functions $f$ that are plurisubharmonic on a domain $\Omega$ and continuous on $\bar{\Omega}$, with functions that are plurisubharmonic on (shrinking) neighborhoods of $\bar{\Omega}$. We show that such approximation is possible if the boundary of $\Omega$ is $C^{0}$ outside a countable exceptional set $E \subset \partial \Omega$. In particular, approximation is possible on the Hartogs triangle. For Hölder continuous $u$, approximation is possible under less restrictive conditions on $E$. We next give examples of domains where this kind of approximation is not possible, even when approximation in the Hölder continuous case is possible.
\end{abstract}

\section{Introduction}

In [Sib] Sibony showed that any bounded $C^{\infty}$-smooth pseudoconvex domain $\Omega$ in $\mathbb{C}^{n}$ has the $P$ (luri)S(ub)H(armonic) Mergelyan property, cf. [Hed]. That is, every function $u$ that is plurisubharmonic on $\Omega$ and continuous on $\bar{\Omega}$ can be approximated uniformly on $\bar{\Omega}$ by continuous plurisubharmonic functions $v_{j}$ that are defined on (shrinking) domains $\Omega_{j}$ that contain $\bar{\Omega}$. Fornæss and the second author extended this result in [FW] to arbitrary domains with $C^{1}$-boundary. Recently, see $[\mathrm{AHP}]$ and [Hed], it was shown that the result remains valid if one only assumes $C^{0}$-boundary.

Recall that a domain $G$ in $\mathbb{R}^{n}$ has $C^{0}$-boundary if $(\bar{G})^{\circ}=G$ and the boundary is locally the graph of a continuous function over an $(n-1)$-dimensional hyperplane. This is equivalent to saying that $G$ has the Segment Property for every $z$ in $\partial G$, imation.

Key words and phrases: plurisubharmonic function, approximation, Mergelyan type approx-

2010 Mathematics Subject Classification: Primary 32U05; secondary 31B25, 31B05. 
cf. [AHP], [Fra] and [Hed], i.e., there exists a neighborhood $U$ of $z$ and a vector $w \in \mathbb{R}^{n}$ such that

$$
U \cap \bar{G}+t w \subset G, \quad \text { for all } 0<t<1 .
$$

For an arbitrary domain in $G$ we will call $z \in \partial G$ a $C^{0}$-boundary point if $G$ has the Segment Property at $z$. The $C^{0}$-boundary points form a relative open subset of $\partial G$.

Let $\Delta$ be the unit disc in $\mathbb{C}$. In $[\mathrm{Hed}]$ it is observed that the domain $\Delta \backslash$ $[-1 / 2,1 / 2]$ does not have the PSH-Mergelyan property. The natural setting to study this kind of approximation problem, however, is the case where $\Omega$ is a fat domain, i.e. $\Omega=(\bar{\Omega})^{o}$. For domains in $\mathbb{C}$ (and in fact $\mathbb{R}^{n}$ ) results from classical potential theory give precise criteria for "Mergelyan type" approximation with harmonic and subharmonic functions. For harmonic functions, this goes already back to Keldysh, [Kel], and Deny, [Den], see also [ArGa], [Gar] and [Hedb]. We recall the result in Section 4, where we give several examples of domains where the PSH-Mergelyan property does not hold.

There is a big gap between the counterexamples in $\mathbb{C}$ and domains with $C^{0}$-boundary. It is a natural question whether the Hartogs triangle, $R=\{(z, w)$ : $0<|z|<|w|<1\}$ has the PSH-Mergelyan property, as it is known that $R$ does not have the Mergelyan property for holomorphic functions. As the main result of this note, however, we will prove the following theorem.

Theorem 2.3. Bounded domains in $\mathbb{C}^{n}$ of which the boundary is $C^{0}$ with the possible exception of a countable set of boundary points, have the PSH-Mergelyan property.

In particular this shows that the Hartogs triangle has the PSH-Mergelyan property.

In Section 3, we study uncountable exceptional sets and show that when extra assumptions are made on the modulus of continuity of the approximand, the result of Theorem 2.3 can be extended to certain uncountable sets. In Section 4 , we give several examples showing the difficulty of characterising the PSH-Mergelyan property. We also make some comparisons to the classical problem of (sub-) harmonic approximation and to approximation involving Hölder continuous functions.

\section{Approximation of plurisubharmonic functions}

Let $\operatorname{PSH}(\Omega)$ denote the plurisubharmonic functions on $\Omega$ and $\operatorname{PSH}(\bar{\Omega})$ the functions that are uniform limits of plurisubharmonic functions defined on (shrinking) neighborhoods of $\bar{\Omega}$. Let $B(a, r) \subset \mathbb{C}^{n}$ denote the ball with center $a$ and radius $r$. 
For the reader's convenience, we recall the familiar definition of modulus of continuity.

Definition 2.1. Suppose that $u$ is a function defined on the set $X$. A function $\omega:[0, \infty] \rightarrow[0, \infty]$ is called a modulus of continuity for $u$ if

$$
|u(z)-u(w)| \leq \omega(|z-w|), \quad \forall z, w \in X
$$

Lemma 2.2. Let $D$ be a bounded domain in $\mathbb{C}^{n}$ and let $u$ be in $\operatorname{PSH}(D) \cap C(\bar{D})$ with modulus of continuity $\omega$. Let $a \in \partial D$. For every small $\varepsilon>0$ there exists a function $v \in \operatorname{PSH}(D) \cup B(a, \varepsilon)$ that is continuous on $\overline{D \cup B(a, \varepsilon)}$ and satisfies

$$
\sup _{\bar{D}}|u-v| \leq 4 \omega\left(\operatorname{diam}(D)^{2 / 3} \varepsilon^{1 / 3}\right) .
$$

Proof. Without loss of generality, assume that $a$ is the origin and that $u(0)=0$.

Let $\eta=\operatorname{diam}(D)^{2 / 3} \varepsilon^{1 / 3}$, and

$$
\alpha=6 \omega(\eta)(\log (\operatorname{diam}(D) / \varepsilon))^{-1}
$$

For $z \in \bar{D}$, define

$$
v(z)= \begin{cases}\max \{u(z)+\alpha \log |z / \eta|,-2 \omega(\eta)\}, & \text { if }|z| \leq \eta \\ u(z)+\alpha \log |z / \eta|, & \text { if }|z|>\eta\end{cases}
$$

Since $u(z)+\alpha \log |z / \eta| \geq-\omega(\eta)$ for $|z|=\eta$ it is obvious that $v \in \operatorname{PSH}(D) \cap C(\bar{D})$. Furthermore, when $|z| \leq \varepsilon$,

$$
\begin{aligned}
u(z)+\alpha \log |z / \eta| & \leq \omega(\varepsilon)+\alpha \log (\varepsilon / \eta) \\
& =\omega(\varepsilon)+\frac{6 \omega(\eta)}{\log (\operatorname{diam}(D) / \varepsilon)} \log (\varepsilon / \eta) \\
& =\omega(\varepsilon)+\frac{6 \omega(\eta)}{\log (\operatorname{diam}(D) / \varepsilon)} \log \left((\varepsilon / \operatorname{diam}(D))^{2 / 3}\right) \\
& \leq \omega(\varepsilon)-4 \omega(\eta) \\
& \leq-3 \omega(\eta),
\end{aligned}
$$

and hence $v \equiv-2 \omega(\eta)$ on $D \cap B(a, \varepsilon)$. Thus setting $v \equiv-2 \omega(\eta)$ extends $v$ as a plurisubharmonic function to $B(a, \varepsilon)$. Furthermore, if $\varepsilon$ is small and $v(z)=u(z)+$ 
$\alpha \log |z / \eta|$, it follows that

$$
\begin{aligned}
|u(z)-v(z)| & \leq \alpha|\log | z / \eta|| \\
& =\alpha|\log | z\left|-\frac{2}{3} \log (\operatorname{diam}(D))-\frac{\log (\varepsilon)}{3}\right| \\
& \leq \frac{2 \alpha}{3}|\log (\operatorname{diam}(D))-\log (\varepsilon)| \\
& =4 \omega(\eta)=4 \omega\left(\operatorname{diam}(D)^{2 / 3} \varepsilon^{1 / 3}\right)
\end{aligned}
$$

and if $v(z)=-2 \omega(\eta)$, it follows that

$$
\begin{aligned}
|u(z)-v(z)| & \leq \omega(\eta)+2 \omega(\eta) \\
& =3 \omega\left(\operatorname{diam}(D)^{2 / 3} \varepsilon^{1 / 3}\right)
\end{aligned}
$$

Theorem 2.3. Let $\Omega$ be a bounded domain in $\mathbb{C}^{n}$. Suppose that $\Omega$ has $C^{0}$ boundary except at a countable set of boundary points $K$. Then $\Omega$ has the PSHMergelyan property.

Proof. Because the set of $C^{0}$-boundary points is open, $K$ is compact. Let $u \in \operatorname{PSH}(\Omega) \cap C(\bar{\Omega})$ and let $K=\left\{a_{1}, a_{2}, \ldots\right\}$. Let $\varepsilon>0$. We apply Lemma 2.2 on a subsequence $\left(a_{n_{j}}\right)$ of $K$ as follows. Applying the lemma to $a_{n_{1}}=a_{1}$ we find a ball $B_{1}=B\left(a_{n_{1}}, r_{1}\right)$ and a continuous function $v_{1}$ defined on the closure of $\Omega_{1}=$ $\Omega \cup B_{1}$ that is plurisubharmonic on $\Omega_{1}$ such that $\left|v_{1}-u\right| \leqslant \varepsilon / 2$ on $\bar{\Omega}$ and such that $K \cap \partial B_{1}=\varnothing$.

Let $a_{n_{2}}$ be the first element of $K$ that is not in $B_{1}$. Then $a_{n_{2}} \notin \overline{B_{1}}$ and applying the lemma gives us a ball $B_{2}$ about $a_{n_{2}}$ and a continuous function $v_{2}$ defined on the closure of $\Omega_{2}=\Omega_{1} \cup B_{2}$ that is plurisubharmonic on $\Omega_{2}$ and satisfies $\left|v_{1}-v_{2}\right| \leqslant 2^{-2} \varepsilon$ on $\overline{\Omega_{1}}$ and such that $\overline{B_{2}} \cap \overline{B_{1}}=\varnothing$ and $K \cap \partial B_{2}=\varnothing$.

We continue in this fashion and obtain a sequence of balls $B_{j}$, and continuous functions $v_{j}$, which are defined on the closure of $\Omega_{j}=\Omega \cup \bigcup_{1}^{j} B_{i}$ and plurisubharmonic on $\Omega_{j}$, such that $\left|v_{j-1}-v_{j}\right|<2^{-j} \varepsilon$ on $\Omega_{j}, K \subset \cup_{j} B_{j}$ and $\overline{B_{i}} \cap \overline{B_{j}}=\varnothing$ for $i \neq j$. Because $K$ is compact, this sequence is finite, $K \subset \cup_{j=1}^{N} B_{j}$ for some $N>0$. The function $v_{N}$ is defined and plurisubharmonic on $\Omega_{N}$ and $\left|v_{N}-u\right|<\varepsilon$ on $\bar{\Omega}$. We will use Gauthier's Localization Theorem, see [Gau], also cf. [Hed], Chapter 5, which states that $f \in C(\bar{\Omega}) \cap \operatorname{PSH}(\bar{\Omega})$ if and only if for every $z_{0} \in \bar{\Omega}$ there is an open set $U$ containing $z_{0}$ such that $f \in C(\overline{\Omega \cap U}) \cap \operatorname{PSH}(\overline{\Omega \cap U})$. We apply this to $v_{N}$. For $z_{0} \in \bar{\Omega} \cap B\left(a_{j}, r_{j}\right)$ or $z_{0} \in \Omega$ this is immediate. If $z_{0} \in \partial \Omega \backslash \cup_{j} B\left(a_{j}, r_{j}\right)$ then $\Omega$ has by assumption the segment property at $z_{0}$. Then there exists a neighborhood $U$ of $z_{0}$ and a vector $w \in \mathbb{C}^{n}$ such that

$$
U \cap \bar{\Omega}+t w \subset \Omega, \quad \text { for all } 0<t<1 .
$$


Fix a ball $B(z, r)$ that is compactly contained in $U$. Then the functions $v_{t}$ defined by $v_{t}(z)=v_{N}(z+t w)$, which are defined on neighborhoods of $\bar{\Omega} \cap B\left(z_{0}, r\right)$, approximate $v_{N}$ uniformly on $\bar{\Omega} \cap \overline{B(z, r)}$ when $t \downarrow 0$. The conclusion is that $v_{N}$, and hence also $u$, belongs to $C(\bar{\Omega}) \cap \operatorname{PSH}(\bar{\Omega})$.

Remark 2.4. Note that this proof also gives a proof of the PSH-Mergelyan property for $C^{0}$-domains. It is shorter, because of Gauthier's theorem, but essentially not different from the proof in $[\mathrm{AHP}]$.

\section{Approximation of Hölder continuous PSH functions}

In many practical cases when working with plurisubharmonic functions, one has some extra quantitative information about the modulus of continuity (see for example $[\mathrm{DF}]$ and $[\mathrm{Sic}]$ ). In such cases we can extend the results of Theorem 2.3 to certain uncountable exceptional sets. In order to describe those sets, we need the following definition.

Definition 3.1. Let $C>1$ and $\varphi: \mathbb{R}^{+} \rightarrow \mathbb{R}^{+}$. The set $K$ satisfies $\operatorname{PS}(C, \varphi)(K$ is said to be $C$-proportionally well separated of negligible $\varphi$-measure), if for each $\varepsilon>0$ there exists a set of balls $\mathcal{F}=\left\{B\left(z_{j}, r_{j}\right)\right\}$ satisfying the following properties:

(1) $\mathcal{F}$ covers $K$;

(2) $\mathcal{F}$ is proportionally $C$-separated in the sense that

$$
B\left(z_{j}, r_{j}\right) \cap B\left(z_{k}, C r_{k}\right)=\varnothing, \quad \text { for all } j \neq k ;
$$

$$
\sum_{j=1}^{\infty} \varphi\left(r_{j}\right)<\varepsilon
$$

Remark 3.2. Note that if $K$ satisfies $\operatorname{PS}(C, \varphi)$, then its generalized Hausdorff measure equals zero, $\Lambda_{\varphi}(K)=0$.

Example 3.3. Let $\mathbf{s}=\left\{s_{1}, s_{2}, \ldots\right\}$ be a sequence of positive numbers $0<s_{j}<1$. Let $I_{0}$ be a closed interval in $\mathbb{R}$ and $C\left(s_{1}\right)$ be $I_{0} \backslash J_{1}$ where $J_{1}$ is an open interval about the center of $I_{0}$ of length $\left(1-s_{1}\right)\left|I_{0}\right|$. Proceeding by induction, $C\left(s_{1}, \ldots, s_{n}\right)$ is obtained by removing from each closed interval $I$ in $C\left(s_{1}, \ldots, s_{n-1}\right)$ an open interval of length $\left(1-s_{n}\right)|I|$ about the center of $I$. The generalized Cantor set $C(\mathbf{s})$ is now defined as follows.

$$
C(\mathbf{s})=\bigcap_{n \geqslant 1} C\left(s_{1}, \ldots . s_{n}\right) .
$$


It is well known that $C(\mathbf{s})$ is homeomorphic to the standard Cantor set, and that its capacity is positive if and only if

$$
\sum_{n=1}^{\infty} \frac{\log s_{n}}{2^{n}}<\infty
$$

cf. $[\mathrm{Nev}]$ and $[\mathrm{Ran}]$.

Now take $\mathbf{s}=\left\{2^{-n}\right\}_{n=1}^{\infty}$. Then since (3.1) is satisfied, $C(\mathbf{s})$ has positive capacity. We claim that for every $\delta>0$ the set $C(\mathbf{s})$ satisfies $\operatorname{PS}(\varphi, C)$ for every $C$ and $\varphi(s)=s^{\delta}$.

Indeed, note that $C\left(2^{-1}, \ldots 2^{-N}\right)$ consists of $2^{N}$ intervals of length $L_{N}=$ $\prod_{1}^{N}\left(s_{j} / 2\right)=2^{-N(N+3) / 2}$. Each of these intervals can be covered by an interval with the same midpoint and radius $r_{N}=L_{N}$. Thus $C(\mathbf{s}) \subset C\left(2^{-1}, \ldots 2^{-N}\right)$ is covered by $2^{N}$ intervals $I_{j}$ of radius $r_{N}$. The distance between $I_{j}$ and $C I_{k}$ is smallest when $I_{j}$ and $I_{k}$ belong to the same interval in $C\left(2^{-1}, \ldots 2^{-(N-1)}\right)$. This distance then equals

$$
L_{N-1}-(2+C) r_{N}=L_{N-1}-(2+C) 2^{-N-1} L_{N-1},
$$

which is positive if $C<2^{N+1}-2$.

For fixed $\delta>0$

$$
\sum_{j=1}^{\infty} r_{j}^{\delta}=2^{N}\left(2^{(-N(N+3)) / 2}\right)^{\delta} \leq 2^{N-\delta\left(N^{2}+3 N\right) / 2},
$$

which tends to 0 if $N \rightarrow \infty$.

The following example shows that it might be hard to give sufficient criteria for sets to be proportionally separated.

Example 3.4. The set $X \subset \mathbb{R}$ defined by $X=\{1 / j: j \in \mathbb{N}\}$ satisfies Property $\operatorname{PS}(C, \varphi)$ with $C=2$ and $\varphi(t)=t^{\alpha}$ for all $\alpha>0$. On the other hand the set $Y=X \cup\{0\}$ does not satisfy Property $\operatorname{PS}\left(C, t^{\alpha}\right)$ for any $C>1$ and $\alpha$.

We are now ready to state and prove the main result of this section.

Theorem 3.5. Suppose that $D \subset \mathbb{C}^{n}$ is a bounded domain and that $u \in$ $\operatorname{PSH}(D) \cap C(\bar{D})$, with a concave modulus of continuity $\omega$. Suppose also that there is a set $E \subset \partial D$ such that each $z \in \partial \Omega \backslash E$ has a neighborhood $U \subset \mathbb{C}^{n}$ such that $u \in \operatorname{PSH}(\overline{U \cap D})$.

If $E$ satisfies $\operatorname{PS}(C, \varphi)$ with $\varphi(t)=\omega(t) \log (1 / t)$, then $u \in \operatorname{PSH}(\bar{D})$.

Remark 3.6. Since $\bar{\Omega}$ is compact and $u$ is continuous, $u$ admits at least one concave modulus of continuity. 
Remark 3.7. One might wonder if the assumption on the modulus of continuity is necessary, or just a consequence of our method of proof. Although we don't know that this condition is sharp, Example 4.3 of the subsequent section shows that some condition on the modulus of continuity is necessary for the theorem to hold.

Proof. Without loss of generality, assume that $\operatorname{diam}(D)=1$. For a fixed $\varepsilon>0$, let $\left\{B\left(z_{j}, r_{j}\right)\right\}_{j=1}^{N}$ be a covering of $K$ satisfying

$$
\sum_{j=1}^{N} \omega\left(r_{j}\right) \log \left(1 / r_{j}\right) \leq \varepsilon
$$

such that $B\left(z_{j}, r_{j}\right) \cap B\left(z_{k}, C r_{k}\right)=\varnothing$ for all $j \neq k$ and some $1<C<2$.

Now let

$$
v_{j}(z)= \begin{cases}\max \left\{u(z)+\frac{2}{\log (C)} \omega\left(C r_{j}\right) \log \left|\frac{z-z_{j}}{C r_{j}}\right|, u\left(z_{j}\right)-\omega\left(C r_{j}\right)\right\}, & \text { if }\left|z-z_{j}\right| \leq C r_{j} \\ u(z)+\frac{2}{\log (C)} \omega\left(C r_{j}\right) \log \left|\frac{z-z_{j}}{C r_{j}}\right|, & \text { if }\left|z-z_{j}\right|>C r_{j}\end{cases}
$$

If $\left|z-z_{j}\right|=C r_{j}$, it follows that

$$
u(z)+2 \omega\left(C r_{j}\right) \log \left|\frac{z-z_{j}}{C r_{j}}\right| \geq u\left(z_{j}\right)-\omega\left(C r_{j}\right),
$$

and therefore it follows by the usual gluing argument that $v \in \operatorname{PSH}(D) \cap C(\bar{D})$. Furthermore, when $\left|z-z_{j}\right| \leq r_{j}$,

$$
\begin{aligned}
u(z)+\frac{2}{\log (C)} \omega\left(C r_{j}\right) \log \left|\frac{z-z_{j}}{C r_{j}}\right| & \leq u\left(z_{j}\right)+\omega\left(r_{j}\right)+\frac{2}{\log (C)} \omega\left(C r_{j}\right) \log \left(r_{j} /\left(C r_{j}\right)\right) \\
& =u\left(z_{j}\right)+\omega\left(r_{j}\right)-\frac{2}{\log (C)} \omega\left(C r_{j}\right) \log (C) \\
& \leq u\left(z_{j}\right)-\omega\left(C r_{j}\right),
\end{aligned}
$$

and hence $v_{j}$ is constant on $D \cap B\left(z_{j}, r_{j}\right)$. Thus $v_{j}$ can be trivially plurisubharmonically extended to $D \cup B\left(z_{j}, r_{j}\right)$.

Now let

$$
\tilde{v}_{j}(z)=v_{j}(z)+\frac{2}{\log (C)} \sum_{k \neq j} \omega\left(C r_{k}\right) \log \left|\frac{z-z_{k}}{C r_{k}}\right|,
$$

and define

$$
v(z)=\max \left\{\tilde{v}_{j}(z): 1 \leq j \leq N\right\} .
$$

We now want to show that $v(z)=\tilde{v}_{j}(z)$ when $\left|z-z_{j}\right|<C r_{j}$, which will imply that $v$ admits a plurisubharmonic extension to $D \cup B\left(z_{j}, r_{j}\right)$. For this, suppose that $\mid z-$ 
$z_{j} \mid<C r_{j}$ and let $k \neq j$ be arbitrary. Then it follows from the fact that $B\left(z_{j}, C r_{j}\right) \cap$ $B\left(z_{k}, C r_{k}\right)=\varnothing$, that

$$
\begin{aligned}
\tilde{v}_{k}(z) & =u(z)+\frac{2}{\log (C)} \sum_{k=1}^{N} \omega\left(C r_{k}\right) \log \left|\frac{z-z_{k}}{C r_{k}}\right| \\
& \leq v_{j}(z)+\frac{2}{\log (C)} \sum_{k \neq j} \omega\left(C r_{k}\right) \log \left|\frac{z-z_{k}}{C r_{k}}\right| \\
& =\tilde{v}_{j}(z) .
\end{aligned}
$$

To see that $v$ approximates $u$, we consider two different cases.

Case 1.

$$
z \in \Omega \cap \bigcup_{j=1}^{N} B\left(z_{j}, C r_{j}\right) .
$$

Suppose that $z \in B\left(z_{j}, C r_{j}\right)$ for some $j=1 \ldots N$. Then it was earlier demonstrated that

$$
v(z)=v_{j}(z)+\frac{2}{\log (C)} \sum_{k \neq j} \omega\left(C r_{k}\right) \log \left|\frac{z-z_{k}}{C r_{k}}\right| .
$$

There are now two possibilities.

Case 1.1. If

$$
v_{j}(z)=u(z)+\frac{2}{\log (C)} \omega\left(C r_{j}\right) \log \left|\frac{z-z_{j}}{C r_{j}}\right|,
$$

then $r_{j} \leqslant\left|z-z_{j}\right| \leqslant C r_{j}$ and hence $\log \left|\frac{z-z_{\ell}}{C r_{\ell}}\right|$ is negative for $\ell=j$ and positive for all other $\ell$. Keeping this in mind and that $\operatorname{diam}(D)<1$, we see that

$$
\begin{aligned}
\tilde{v}_{j}(z)-u(z) & =\frac{2}{\log (C)} \sum_{k=1}^{N} \omega\left(C r_{k}\right) \log \left|\frac{z-z_{k}}{C r_{k}}\right| \\
& \leq \frac{2}{\log (C)} \sum_{k \neq j} \omega\left(C r_{k}\right) \log \left(\frac{1}{C r_{k}}\right) \\
& \leq \frac{2}{\log (C)} \sum_{k \neq j} \omega\left(C r_{k}\right) \log \left(\frac{1}{r_{k}}\right) \\
& \leq \frac{2 C}{\log (C)} \sum_{k \neq j} \omega\left(r_{k}\right) \log \left(\frac{1}{r_{k}}\right) \\
& \leq \frac{2 C}{\log (C)} \varepsilon
\end{aligned}
$$

where we in the second to last inequality have used the fact that $\omega$ is concave. 
On the other hand, it also holds that

$$
\begin{aligned}
\tilde{v}_{j}(z)-u(z) & =\frac{2}{\log (C)} \sum_{k=1}^{N} \omega\left(C r_{k}\right) \log \left|\frac{z-z_{k}}{C r_{k}}\right| \\
& \geq \frac{2}{\log (C)} \omega\left(C r_{j}\right) \log \left|\frac{z-z_{j}}{C r_{j}}\right| \\
& \geq \frac{2}{\log (C)} \omega\left(C r_{j}\right) \log \left(\frac{1}{C}\right) \\
& =-2 \omega\left(C r_{j}\right) \geq-2 C \omega\left(r_{j}\right) \geq-4 \varepsilon
\end{aligned}
$$

Case 1.2. If $v_{j}(z)=u\left(z_{j}\right)-\omega\left(C r_{j}\right)$, it similarly follows that

$$
\begin{aligned}
u(z)-\tilde{v}_{j}(z) & \leq u(z)-u\left(z_{j}\right)+\omega\left(C r_{j}\right)-\frac{2}{\log (C)} \sum_{k \neq j} \omega\left(C r_{k}\right) \log \left|\frac{z-z_{k}}{C r_{k}}\right| \\
& \leq 2 \omega\left(C r_{j}\right) \leq 2 C \varepsilon
\end{aligned}
$$

and

$$
\begin{aligned}
\tilde{v}_{j}(z)-u(z) & \leq u\left(z_{j}\right)-u(z)-\omega\left(C r_{j}\right)+\frac{2}{\log (C)} \sum_{k \neq j} \omega\left(C r_{k}\right) \log \left|\frac{z-z_{k}}{C r_{k}}\right| \\
& \leq \omega\left(C r_{j}\right)-\omega\left(C r_{j}\right)+\frac{2}{\log (C)} \sum_{k \neq j} \omega\left(C r_{k}\right) \log \left(1 / C r_{k}\right) \\
& \leq \frac{2 C}{\log (C)} \sum_{k \neq j} \omega\left(r_{k}\right) \log \left(1 / r_{k}\right) \\
& \leq \frac{2 C}{\log (C)} \varepsilon .
\end{aligned}
$$

Case 2.

$$
z \in \Omega \backslash \bigcup B\left(z_{j}, C r_{j}\right)
$$

In this case note that now for all $j$ it holds that $v_{j}(z)=u(z)+\frac{2}{\log (C)} \omega\left(C r_{j}\right) \log \left|\frac{z-z_{j}}{C r_{j}}\right|$. It follows similarly as above that

$$
\begin{aligned}
\left|u(z)-\tilde{v}_{j}(z)\right| & =\frac{2}{\log (C)} \sum_{k=1}^{N} \omega\left(C r_{k}\right) \log \left|\frac{z-z_{k}}{C r_{k}}\right| \\
& \leq \frac{2 C}{\log (C)} \sum_{k=1}^{N} \omega\left(r_{k}\right) \log \left(\frac{1}{r_{k}}\right) \\
& \leq \frac{2 C}{\log (C)} \varepsilon .
\end{aligned}
$$


We have thus shown that on $\bar{\Omega}, u$ is a uniform limit of functions like $v$. To show that $u \in \operatorname{PSH}(\bar{D})$, it therefore suffices to show that $v \in \operatorname{PSH}(\bar{D})$. It follows from Gauthier's localization lemma, that it is enough to show that every $z \in \partial D$ has a neighborhood $U_{z}$ such that $v \in \operatorname{PSH}\left(\overline{U_{z} \cap D}\right)$. For $z \in \partial D \backslash K$, this follows from the assumptions of the theorem and for $z \in K$, it follows by the construction that there is a neighborhood $U_{z}$ of $z$ where $v$ is constant and hence trivially in $\operatorname{PSH}\left(\overline{U_{z} \cap D}\right)$.

\section{Domains without the Mergelyan property}

The following result, which was mentioned in the introduction, goes back to Keldysh and Deny in case of harmonic functions and is in full generality a consequence of results by Bliedtner and Hansen, [BlHa], and cf. [Gar]. It reads as follows.

Theorem 4.1. Let $K$ be a compact subset of $\mathbb{R}^{n}$. The following are equivalent

(1) Every function $u$ that is continuous on $K$ and (sub)harmonic on $K^{o}$ can be uniformly approximated on $K$ by functions that are (sub)harmonic on (shrinking) neighborhoods of $K$.

(2) The sets $\mathbb{R}^{n} \backslash K$ and $\mathbb{R}^{n} \backslash K^{o}$ are thin at the same points.

With this result, examples like the following have been constructed.

Example 4.2. Let $\left\{a_{j}\right\}$ be a sequence in $\Delta \backslash[-1 / 2,1 / 2]$, such that $\overline{\left\{a_{j}\right\}}=\left\{a_{j}\right\} \cup$ $[-1 / 2,1 / 2]$. Define $c_{j}>0$ sequentially such that $h(z)=\sum_{j} c_{j} \log \left|z-a_{j}\right|>-1$ on $[-1 / 2,1 / 2]$, and next $r_{j}$ in such a way that $h<-2$ if $\left|z-a_{j}\right|<r_{j}$ and, moreover the discs $\left\{\left|z-a_{j}\right| \leqslant r_{j}\right\}$ are disjoint. Then

$$
D=\Delta \backslash \overline{\left(\cup_{j}\left\{\left|z-a_{j}\right|<r_{j}\right\}\right)}=\Delta \backslash\left([-1 / 2,1 / 2] \cup \bigcup_{j}\left\{\left|z-a_{j}\right| \leqslant r_{j}\right\}\right)
$$

is a fat domain in $\mathbb{C}, K=\bar{D}$, and $K^{o}=D$ do not satisfy condition (2). In fact $\mathbb{C} \backslash K=\bigcup_{j}\left\{\left|z-a_{j}\right|<r_{j}\right\}$ is thin at all points in $[-1 / 2,1 / 2]$, but $\mathbb{C} \backslash K^{o}$ is not. Hence $K$ does not have the approximation property (1).

For completeness, and because Theorem 4.1 was misunderstood in [HP] see also [Hed, chapter 6], we show directly that $D$ is hyperconvex, but not $P$-hyperconvex, that is, $D$ admits a bounded subharmonic exhaustion function $g$, but no such function belongs to $\operatorname{PSH}(\bar{D})$. This corrects Remark 4.10 in [HP].

Observe that the domain $D$ is regular for the Dirichlet problem, since it has no isolated boundary points. Denote by $F$ the solution of the Dirichlet problem on $D$ with boundary values $|z|^{2}$ on $\partial D$. Then the function $z \mapsto|z|^{2}-F(z)$ is a bounded 
subharmonic exhaustion function. To see that no bounded subharmonic exhaustion function $g$ can be in $\operatorname{PSH}(K)$, notice that $[-1 / 2,1 / 2]$ is contained in the fine interior of $K$, because $[-1 / 2,1 / 2] \subset\{h>-3 / 2\}$, a finely open subset of $K$. Now if $g$ were in $\operatorname{PSH}(K), g$ would be finely subharmonic on the fine interior of $K$ as a uniform limit of subharmonic functions. But $g=0$ on $[-1 / 2,1 / 2]$ and $\leqslant 0$ on a fine neighborhood of $K$, which would contradict the fine maximum principle, cf. [Fug, Theorem 12.6].

Observe that this also shows that $D$ does not have the PSH-Mergelyan property either.

The example in [Hed], mentioned in the introduction, can be adapted to show that even if the exceptional set $K$ in the boundary is very small, the domain will in general not have the PSH-Mergelyan property.

Example 4.3. (1) Let $C=C(\mathbf{s})\left(\mathbf{s}=\left\{2_{k \geqslant 1}^{-k}\right\}\right)$ in the interval $[-1 / 2,1 / 2]$ be the Cantor set of Example 3.3. We have seen that $C$ has Hausdorff dimension 0 and positive capacity. Let $\Delta$ be the open disc with radius 2 in $\mathbb{C}$, and let $D=\Delta \backslash C$. Then $D$ is regular for the Dirichlet problem, and hence there exists a continuous function $u$ on $\bar{\Delta}$ that is harmonic on $D$ satisfying $\left.u\right|_{\{|z|=2\}}=0$ and $\left.u\right|_{C}=1$. Observe that $u \notin \operatorname{PSH}(\bar{D}) \cap C(\bar{D})$ because of the maximum principle.

As $D$ is a regular domain for the Dirichlet problem, it admits a bounded continuous subharmonic exhaustion function $\psi$ with $-1 \leqslant \psi \leqslant 0$. We set

$$
\Omega=\{(z, w) \in D \times \mathbb{C}:|w|+\psi(z)<0\}
$$

By its definition $\Omega$ is hyperconvex and fat. It is $C^{0}$ at all boundary points of the form $\left(z, \psi(z) e^{i \theta}\right), z \in D, \theta \in[0,2 \pi)$. The function $u$, now viewed as a function on $\bar{\Omega}$, is not in $\operatorname{PSH}(\bar{\Omega}) \cap C(\bar{\Omega})$, again because of the maximum principle.

In fact, the functions $u$ and $\psi$ in the example cannot even be approximated by subharmonic functions that are Hölder continuous on $\Delta$. Indeed, by a theorem of Sadullaev and Yarmetov, [SaYa], such Hölder continuous functions would in fact be subharmonic on $\Delta$, again violating the maximum principle.

Similarly, we find that with $\Omega$ and $u$ as above, $u$ is not a uniform limit on $\bar{\Omega}$ of plurisubharmonic functions on $\Omega$ that are Hölder continuous on $\bar{\Omega}$. On the other hand, by Theorem 3.5, every Hölder continuous function in $\operatorname{PSH}(\Omega)$ belongs to $\operatorname{PSH}(\bar{\Omega})$.

In view of Theorem 4.1 and Example 4.2 one might hope that e.g. conditions like: the sets $\mathbb{C}^{n} \backslash K$ and $\mathbb{C}^{n} \backslash K^{o}$ are (pluri-) thin at the same points, or perhaps that $K \cap L$ has the PSH-Mergelyan property for all complex lines $L$, would be equivalent with the PSH-Mergelyan property. This is not the case. 
Example 4.4. Let $K=\{(z, w): z \in \bar{\Delta},|w| \leqslant d(z,[-1 / 2,1 / 2])\}$ then $\mathbb{C}^{2} \backslash K$ and $\mathbb{C}^{2} \backslash$ $K^{o}$ are both thin and pluri-thin at points of $K^{o}$ and not thin or pluri-thin at other points of $\mathbb{C}^{2}$. For points in $K \backslash K^{o}$ this follows from the Poincaré Zaremba Criterion: in such points $p$ there exists an open solid cone of revolution with vertex $p$ in $\mathbb{C}^{2} \backslash K$, hence for a (pluri-) subharmonic function $h$ defined in a neighborhood of $p$ we have $\lim \sup _{z \rightarrow p, z \notin K} h(z)=h(p)$. However, $K$ does not have the PSH-Mergelyan property.

Example 4.5. Let $\Omega$ be the domain of Example 4.3, we will write $w=u+i v$, and let $\widetilde{\Omega}=(\Omega \cup\{v>0\}) \cap\left\{|z|^{2}+|w|^{2}<3 / 2\right\}$. Then $\partial \widetilde{\Omega}$ has $C^{0}$ boundary. This is clear at boundary points $(z, w)$ with $w \neq 0$ and at boundary points belonging to $\left\{|z|^{2}+|w|^{2}=3 / 2\right\}$. Define for $z \in \Delta$ and $|u|+\varphi(z) \leqslant 0$ the continuous function $\psi$ : $(z, u) \mapsto-\sqrt{\varphi^{2}(z)-u^{2}}$. Then the part of the boundary where $|z|^{2}<3 / 2$ and $v \leqslant 0$ is the graph $(z, u+i \psi(z, u))$ over a suitable domain in $D \times \mathbb{R}$. Hence $\widetilde{\Omega}$ has the PSH-Mergelyan property, but its intersection with the line $w=0$ has not.

Question 4.6. With an eye to Example 4.3 we ask the following. Suppose a domain $\Omega$ has $C^{0}$-boundary, except for a compact set $K$ in $\partial \Omega$. Suppose also that $K$ is a polar subset of a 1-dimensional analytic variety $J$. Does $\Omega$ have the PSH-Mergelyan property?

Question 4.7. Suppose that $\Omega$ is a fat, bounded, contractible domain. Does $\Omega$ have the PSH-Mergelyan property?

\section{References}

[ArGa] Armitage, D. H. and Gardiner, S. J., Classical Potential Theory, Springer Monographs in Mathematics, Springer-Verlag London, Ltd., London, 2001.

[AHP] Avelin, B., Hed, L. and Persson, H., Approximation of plurisubharmonic functions, Complex Var. Elliptic Equ. 61 (2016), 23-28.

[BlHa] Bliedtner, J. and Hansen, W., Simplicial cones in potential theory. II. Approximation theorems, Invent. Math. 46 (1978), 255-275.

[Den] Deny, J., Systèmes totaux de fonctions harmoniques, Ann. Inst. Fourier (Grenoble) 1 (1949, 1950), 103-113.

[DF] Diederich, K. and Fornæss, J. E., Pseudoconvex domains: bounded strictly plurisubharmonic exhaustion functions, Invent. Math. 39 (1977), 129-141.

[FW] Fornæss, J. E. and Wiegerinck, J., Approximation of plurisubharmonic functions, Ark. Mat. 27 (1989), 257-272.

[Fra] Fraenkel, L. E., On regularity of the boundary in the theory of Sobolev spaces, Proc. Lond. Math. Soc. 39 (1979), 385-427.

[Fug] Fuglede, B., Finely Harmonic Functions, Springer LNM 289, 1972.

[Gar] Gardiner, S. J., Harmonic Approximation, London Mathematical Society Lecture Note Series 221, Cambridge University Press, Cambridge, 1995. 
[Gau] Gauthier, P., Approximation by (pluri)subharmonic functions: fusion and localization, Canad. J. Math. 44 (1992), 941-950.

[Hed] Hed, L., The Plurisubharmonic Mergelyan Property, Thesis, Umeå, 2012.

[HP] Hed, L. and Persson, H., Plurisubharmonic approximation and boundary values of plurisubharmonic functions, J. Math. Anal. Appl. 413 (2014), 700-714.

[Hedb] Hedberg, L. I., Approximation by harmonic functions, and stability of the Dirichlet problem, Expo. Math. 11 (1993), 193-259.

[Kel] Keldysh, M. V., On the solubility and the stability of Dirichlet's problem, Uspekhi Mat. Nauk 8 (1941), 171-231 (in Russian).

[Nev] Nevanlinna, R., Analytic Functions, Grundl. Math. Wiss. 162, 1970.

[Ran] Ransford, T., Potential Theory in the Complex Plane, London Math. Soc. Student Texts 28, 1995.

[SaYa] Sadullaev, A. and Yarmetov, Zh. R., Removable singularities of subharmonic functions in the class $\operatorname{Lip}_{\alpha}$, Mat. Sb. 186 (1995), 131-148 (in Russian), translation in Sb. Math. 186 (1995), 133-150.

[Sib] Sibony, N., Une classe de domaines pseudoconvexes [A class of pseudoconvex domains], Duke Math. J. 55 (1987), 299-319.

[Sic] Siciak, J., Wiener's type sufficient conditions in $\mathbb{C}^{N}$, Univ. Iagel. Acta Math. 35 (1997), 47-74.

Håkan Persson

Department of Mathematics

Uppsala University

SE-75106

Uppsala

Sweden

haakanpersson@gmail.com
Jan Wiegerinck

KdV Institute for Mathematics

University of Amsterdam

Science Park 105-107

P.O. Box 94248, $1090 \mathrm{GE}$

Amsterdam

The Netherlands

j.j.o.o.wiegerinck@uva.nl

Received October 5, 2016

in revised form January 27, 2017 